\title{
Recombination between the mouse $Y$ chromosome short arm and an additional $Y$ short arm-derived chromosomal segment attached distal to the $\mathrm{X}$ chromosome PAR
}

\author{
Fanny Decarpentrie $^{1,2}$ - Obah A. Ojarikre ${ }^{1,2} \cdot$ Michael J. Mitchell ${ }^{3}$ Paul S. Burgoyne ${ }^{1,2}$
}

Received: 1 May 2015 /Revised: 9 November 2015 / Accepted: 11 November 2015 /Published online: 23 November 2015

(C) The Author(s) 2015. This article is published with open access at Springerlink.com

\begin{abstract}
In a male mouse, meiosis markers of processed DNA double strand breaks (DSBs) such as DMC1 and RAD51 are regularly seen in the non-PAR region of the $\mathrm{X}$ chromosome; these disappear late in prophase prior to entry into the first meiotic metaphase. Marker evidence for DSBs occurring in the non-PAR region of the $\mathrm{Y}$ chromosome is limited. Nevertheless, historically it has been documented that recombination can occur within the mouse Y short arm (Yp) when an additional Yp segment is attached distal to the $\mathrm{X}$ and/ or the Y pseudoautosomal region (PAR). A number of recombinants identified among offsprings involved unequal exchanges involving repeated DNA segments; however, equal exchanges will have frequently been missed because of the paucity of markers to differentiate between the two Yp segments. Here, we discuss this historical data and present extensive additional data obtained for two mouse models with $\mathrm{Yp}$ additions to the X PAR. PCR genotyping enabled
\end{abstract}

This article is part of a Special Issue on "Recent advances in meiotic chromosome structure, recombination and segregation" edited by Marco Barchi, Paula Cohen and Scott Keeney

Electronic supplementary material The online version of this article (doi:10.1007/s00412-015-0559-0) contains supplementary material, which is available to authorized users.

Fanny Decarpentrie

Fanny.Decarpentrie@crick.ac.uk

1 Department of Stem Cell Biology and Developmental Genetics, MRC National Institute for Medical Research, The Ridgeway, Mill Hill, London NW7 1AA, UK

2 Mill Hill Laboratory, The Francis Crick Institute, London NW7 1AA, UK

3 Inserm UMR_S 910, Aix Marseille Université, 13005 Marseille, France identification of a wider range of potential recombinants; the proportions of Yp exchanges identified among the recombinants were 9.7 and $22.4 \%$. The frequency of these exchanges suggests that the Yp segment attached to the $\mathrm{X}$ PAR is subject to the elevated level of recombinational DSBs that characterizes the PAR.

Keywords Mouse $\cdot$ Meiosis $\cdot$ Y-chromosome $\cdot$ Short arm (Yp) $\cdot$ Recombination $\cdot$ Spo11

\section{Introduction}

In a normal male mouse meiosis, the regular Spo11-dependent recombination that occurs between the $\mathrm{X}$ and $\mathrm{Y}$ chromosomes is efficiently restricted to their PAR regions (Burgoyne 1982; Kauppi et al. 2011), although X-Y synapsis may spread beyond the PAR boundary during the mid pachytene stage. The initial synapsis of the PARs protects the PAR chromatin from meiotic transcriptional silencing that is initiated at the zygotene/pachytene transition (reviewed by Burgoyne et al. 2009), but it is not widely appreciated that the subsequent spreading of synapsis may well interfere with the transcriptional silencing of X-Y chromatin proximal to the PARs. This is likely the explanation for the occasional finding of pachytene spermatocytes transcribing the X-linked gene $\mathrm{Scml} 2$ (Royo et al. 2010), since it is located only $\sim 7 \mathrm{Mb}$ from the X PAR (total X length $\sim 177 \mathrm{Mb}$ ).

Although immunostaining for proteins that localize to sites of DSBs (e.g. RAD51, DMC1, RPA) have shown that the X has multiple DSBs that could enable recombination with a synapsed non-PAR Y segment lacking DSBs (Kauppi et al. 2011, 2012; Moens et al. 1997; Plug et al. 1998), translocations between the $\mathrm{X}$ and the $\mathrm{Y}$ are extremely rare. This is presumably because regions of homology are very limited 
and unlikely to be juxtaposed during the presynaptic homology search. This is supported by the sequence data for multicopy $\mathrm{X}$ and $\mathrm{Y}$ paralogs that indicate crossovers leading to exchange between $\mathrm{X}$ and $\mathrm{Y}$ copies are very rare despite substantial homology (Soh et al. 2014). Markers of DSBs have rarely been reported on the non-PAR Y, but it can participate in homology-driven recombination as evidenced by mouse and human Y chromosome sequence data, which implicate intrachromatid or inter-sister recombination between ampliconic repeats as responsible for the generation of rare $\mathrm{Y}$ deletions and rearrangements (Lange et al. 2009, 2013; Skaletsky et al. 2003; Soh et al. 2014). It is therefore reasonable to posit that rare DSBs in Yp could participate in rare recombination with a synaptic partner with substantial homology, provided they are juxtaposed during the presynaptic homology search.

That this may be the case is supported by the historical identification of occasional $\mathrm{Y}$ short arm recombinants among the progeny of males in which there was a $\mathrm{Y}$ short arm (Yp) derivative $\left(S x r^{a}\right.$ or $S x r^{b}$-see Fig. 1b, d) attached distal to the X PAR, or distal to the $\mathrm{X}$ and $\mathrm{Y}$ PARs (Epplen et al. 1988; Laval et al. 1995; McLaren et al. 1988, 1992; Simpson et al. 1984). While there was the expected frequent transfer of the complete $S x r$ segment from the Y PAR to the X PAR (Fig. 1c) or vice versa, there were also exchanges of partial $S x r$ segments with Yp; the latter exchanges must involve DSBs located within Sxr or within Yp. These partial Sxr exchanges could be balanced exchanges or unbalanced exchanges; the latter are an expected consequence of the presence of substantial regions of sequence repeats on the mouse $\mathrm{Y}$ short arm (Soh et al. 2014).

Two of these studies are particularly relevant in the context of the new data reported here. Firstly, Simpson et al. (1984) identified among the progeny of an XSxrYSxr male (with $S x r$ attached to the X and Y PARs) a mouse carrying an $S x r$ deletion variant that was designated $S x r^{b}$ (with the original $S x r$ then being designated $S x r^{a}$ ). Subsequent work established that the deletion resulted from a crossover located within $Z f y l$ of the Y-located $S x r$ and within Zfy2 of the X-located $S x r$, resulting in a $1.38-\mathrm{Mb}$ deletion removing six Yp genes and creating a Zfy2/1 fusion gene (Fig. 1d) (Decarpentrie et al. 2012; Mazeyrat et al. 1998; Simpson and Page 1991). Secondly, McLaren et al. (1992) analyzed the progeny of XSxr ${ }^{\text {b- }}$ $\mathrm{YSxr}^{a}$ males that enabled them to document exchanges between $S x r^{a}$ and $S x r^{b}$ and between $S x r^{b}$ and Yp. Significantly, the $S x r^{a}-S x r^{b}$ exchanges must have involved DSBs located within these Yp derivatives attached to the PARs. Here, we report a strikingly high frequency of $S x r^{b}-Y p$ recombination in two mouse models that have $\mathrm{Sxr}^{b}$ attached to the X PAR; we propose that this high frequency of DSBs is a consequence of the spreading of DSB hotspot activity from the X PAR into $\mathrm{Sxr}^{b}$.

\section{Materials and methods}

\section{Mouse breeding}

Mice were produced on an outbred albino MF1 (NIMR stock) background. $\mathrm{XSxr}^{b} \mathrm{Y}$ males (Fig. 2a) were generated by mating $\mathrm{XYSxr}^{b}$ males $\left(\mathrm{Sxr}^{b}\right.$ attached to the $\mathrm{Y}$ $\mathrm{PAR})$ to females carrying the $\mathrm{X}$-autosome translocation $\mathrm{T}(\mathrm{X} ; 16) 16 \mathrm{H}$ (T16H/X females). PCR genotyping for $S x r^{b}$ markers was then used to identify $\mathrm{T} 16 \mathrm{H} / \mathrm{XS} x r^{b}$ mice that have developed as females-these mice can be female despite the presence of $S r y$ in $S x r^{b}$, because the presence of the $\mathrm{T} 16 \mathrm{H}$ translocation ensures that the inactive $\mathrm{X}$ is always the $\mathrm{X}$ carrying $\mathrm{Sxr}^{b}$ (Cattanach et al. 1982; McLaren and Monk 1982). The T16H/XSxr${ }^{b}$ females were then mated to $X Y$ males, and their $X \mathrm{Sxr}^{b} Y$ sons were identified as fertile males (male T16H carriers are sterile) positive for $\mathrm{Sxr}^{b}$ (PCR Sxr , Table 2). $X \mathrm{Sxr}^{b} Y^{*}$ males (Fig. 3a) were originally generated for another study (Vernet et al. 2014). They were produced in the same way as $X \mathrm{Sxr}^{b} Y$ males except that the T16H/XSxr females were mated to $X Y^{*}$ males (Burgoyne et al. 1998; Eicher et al. 1991).

The $\mathrm{XSxr}^{b} \mathrm{Y}$ and $\mathrm{XSxr} r^{b} \mathrm{Y}^{*}$ males were mated to $\mathrm{XX}$ females in order to produce the progeny to be screened for recombinants. In most cases, the females mated to $\mathrm{XSxr}^{b} \mathrm{Y}^{*}$ males were homozygous for the $\mathrm{X}$-linked coat marker Patchy fur (Paf) since this provides an independent check on some of the results obtained from the PCR genotyping (see below). Importantly, it enables the positive identification of XO female progeny as hemizygous Paf females, which are known to be produced at an elevated frequency when the $\mathrm{Y}^{*}$ chromosome is present (Burgoyne and Evans 2000; Eicher et al. 1991).

\section{Screening for sex chromosomally recombinant offspring (Tables 1, 2)}

The initial screen was based on phenotypic markers:

1. Fur phenotype (when the mothers were Paf homozygotes) A few $\mathrm{XSxr} r^{b} \mathrm{Y}$ males and nearly all $\mathrm{XSxr} r^{b} \mathrm{Y}^{*}$ males were mated to females homozygous for the X-linked Patchy fur (Paf) mutation (Lane and Davisson 1990). At 10-15 days old, three fur phenotypes are clearly distinguishable (Burgoyne and Evans 2000) that are independent of their $\mathrm{Yp} / \mathrm{Sxr}$ status: wild type (wt) that identifies $\mathrm{X}^{\mathrm{Paf}} \mathrm{Y}^{*}$ in which the effect of the Paf mutation is masked by the presence of the wild type allele on the $\mathrm{Y}^{*}$ chromosome, "patchy fur" $(\mathrm{Paf} /+)$ due to the very sparse hair in regions where the $\mathrm{X}^{P a f}$ is expressed as in $\mathrm{X}^{P a f} \mathrm{X}$ and $\mathrm{X}^{P a f} \mathrm{X}^{\mathrm{Y}}$ ), 


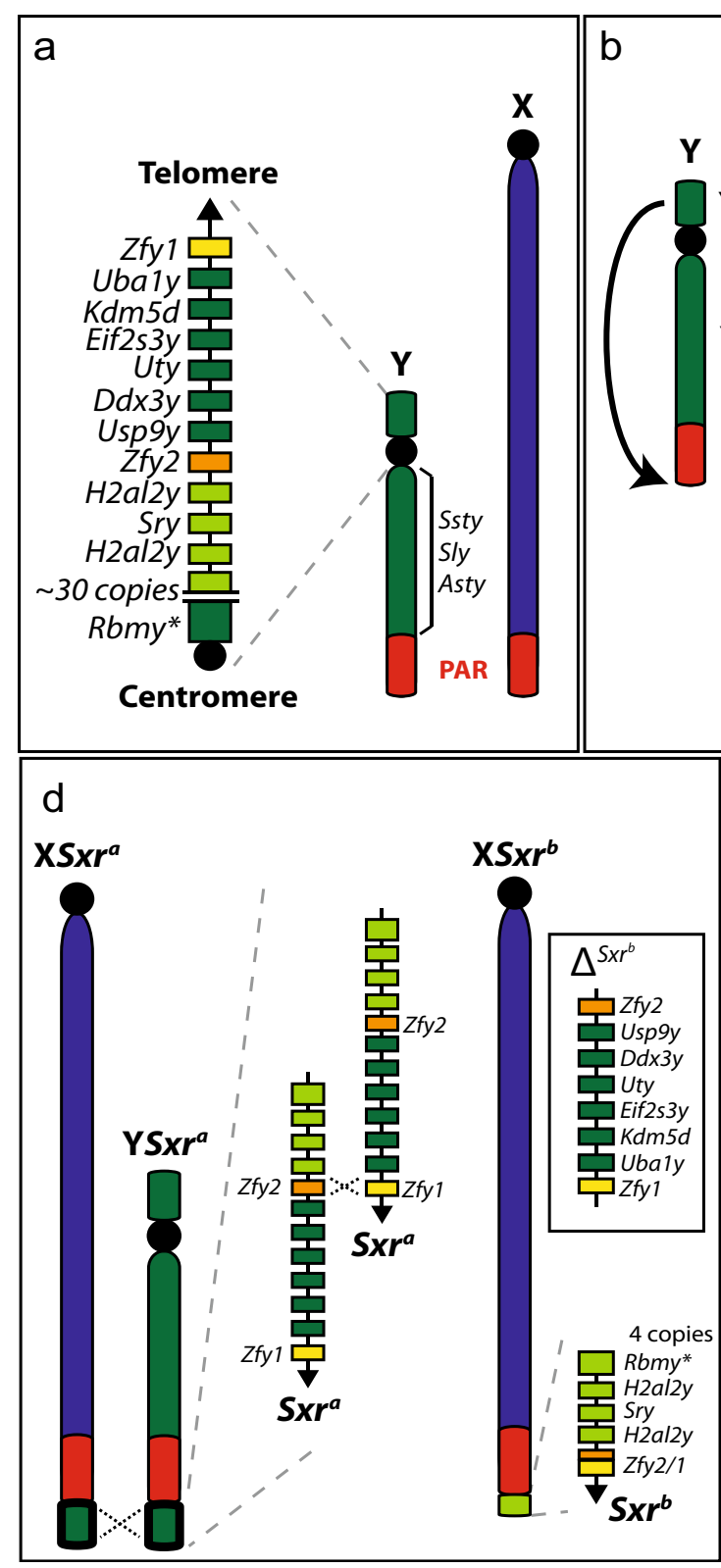

Fig. 1 Origin of $S x r$ variants. (a) Wild type XY. (b) Duplication and translocation of Yp created $S x r^{a}$. (c) PAR-PAR recombination in XYSxr${ }^{a}$ males generates the $\mathrm{XSxr}{ }^{a}$ chromosome. (d) An unequal crossover in an $X \mathrm{Sxr}^{a}{ }^{\mathrm{YSSx}}{ }^{a}$ male created the Sxr ${ }^{b}$ deletion variant. (Color codes: Red $=$

almost nude (Paf) due to the absence of a paternally derived $\mathrm{X}$ as in $\mathrm{X}^{\mathrm{Paf}} \mathrm{O}$.

2. Gender Based on external examination at weaning.

3. Testis size Small 15-26-mg/testis (S) that is associated with the presence of two $\mathrm{X}$ chromosomes and/or with the absence of the spermatogonial proliferation gene Eif2s3y that is deleted in $\mathrm{Sxr}^{b}$; Medium/Large 70-120$\mathrm{mg} /$ testis $(\mathrm{M} / \mathrm{L})$.

PCR analysis using DNA samples was used to confirm and extend diagnoses of sex chromosome complement based on phenotypic markers. They were also used to detect $\mathrm{Yp} / S x^{b}$
PAR; Blue $=$ non-PAR X; Dark green $=$ non-PAR Y except $S x r^{b}$; Light green $=S x r^{b} ;$ Yellow $=Z$ Zfy1; Orange $=Z$ Zfy2; Orange $/$ Yellow $=Z$ Zfy $2 / 1$ fusion gene.) * Rbmy copy number estimates based on information provided by Soh et al. (2014) and Mahadevaiah et al. (1998)

recombinants based on sequence information for $\mathrm{Sxr}^{b}$ and Yp (Decarpentrie et al. 2012; Mazeyrat et al. 1998; Soh et al. 2014); these were designed to enable the detection of recombination events in homologous regions that would be expected to promote synapsis when juxtaposed during the homology search phase (Supplemental Fig. S1). Primer sequences are in Table 2.

1. PCR Sxr ${ }_{-}^{b}$ (Decarpentrie et al. 2012) The $Z f y 2 / 1$ junction fragment was PCR-amplified using primers o3452/ 03072. The amplified fragment was then digested using 
Fig. 2 Recombination in $\mathrm{XSxr} r^{b} \mathrm{Y}$ males. (a) PAR-PAR recombination. (b) $\mathrm{Yp}-\mathrm{Sx} r^{b}$ recombination with expanded views showing the $\mathrm{Y}$ gene content of the paired segments and of the two types of recombinant from crossover 1 (black cross); (c) Bar chart of nonrecombinant (No) and recombinant (Yp-Sxr ${ }^{b}$, PAR-PAR) frequencies

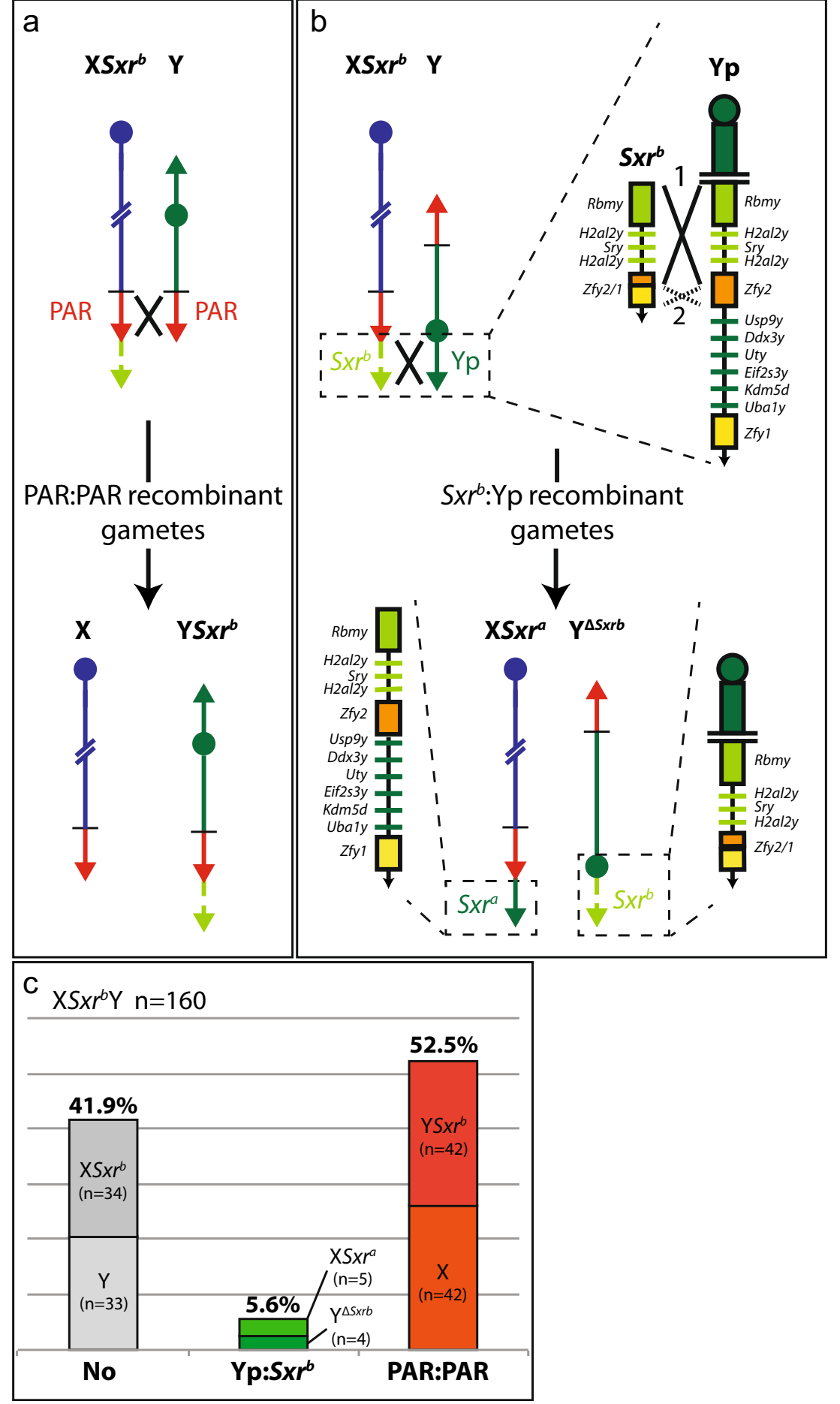

the restriction enzyme RsaI generating a 224 bp $S x r^{b}$-specific fragment.

2. PCR Yp To detect the presence of Yp or $S x r^{a}$, we used primers Smc1/Smc2 that amplify a 300-bp fragment of Yp-linked $K d m 5 D$ (aka $S m c y$ ) and a 330-bp fragment of $\mathrm{X}$-linked $K d m 5 C$ (aka $S m c x$ ) that acts as an amplification control.
3. $P C R Y q$ To detect the presence of the long arm of the $\mathrm{Y}$ (Yq), we used primers PC11fp2F and R that amplify a 237-bp fragment of Ssty2 (present in multiple copies) and primers Sstxfp10 and Sstxrp13 that amplify a 350-bp fragment of X-linked Sstx (present in multiple copies-Soh et al. 2014) that acts as an amplification control. 
Fig. 3 Recombination in $\mathrm{XSxr}{ }^{b} \mathrm{Y}^{*}$ males. (a) The sex chromosomes of $\mathrm{XSxr}^{b} \mathrm{Y}^{*}$ males showing the complex $\mathrm{Y}^{*} \mathrm{PAR}$ and associated $\mathrm{X}$-derived centromere, note that the original Y centromere is inactive. (b) PAR-PAR recombination arising from parasynapsed and staggered associations - note that the dicentric $\mathrm{X}$ and $\mathrm{Y} S x r^{b}$ (with inactive $\mathrm{Y}$ centromere) recombinant chromosomes are prone to loss at $\mathrm{MI}$ thus generating " $\mathrm{O}$ " gametes and are not present in the offspring. (c) Yp-Sxr $r^{b}$ recombination with expanded views showing the $\mathrm{Y}$ gene content of the paired segments and potential crossovers - no recombinants were obtained from crossover 2 , and recombinants from crossover 4 could not be detected due to a lack of markers. (d) The four types of recombinant from crossovers 1 and 3. (e) Bar chart of non-recombinant and recombinant frequencies. "Other $n=4$ " comprises one animal from an $\mathrm{X} \Delta S x r^{\mathrm{b}}$ gamete and three from $\mathrm{Y}^{* \Delta \Delta S x \mathrm{ra}}$ gametes (generated by crossover 3)

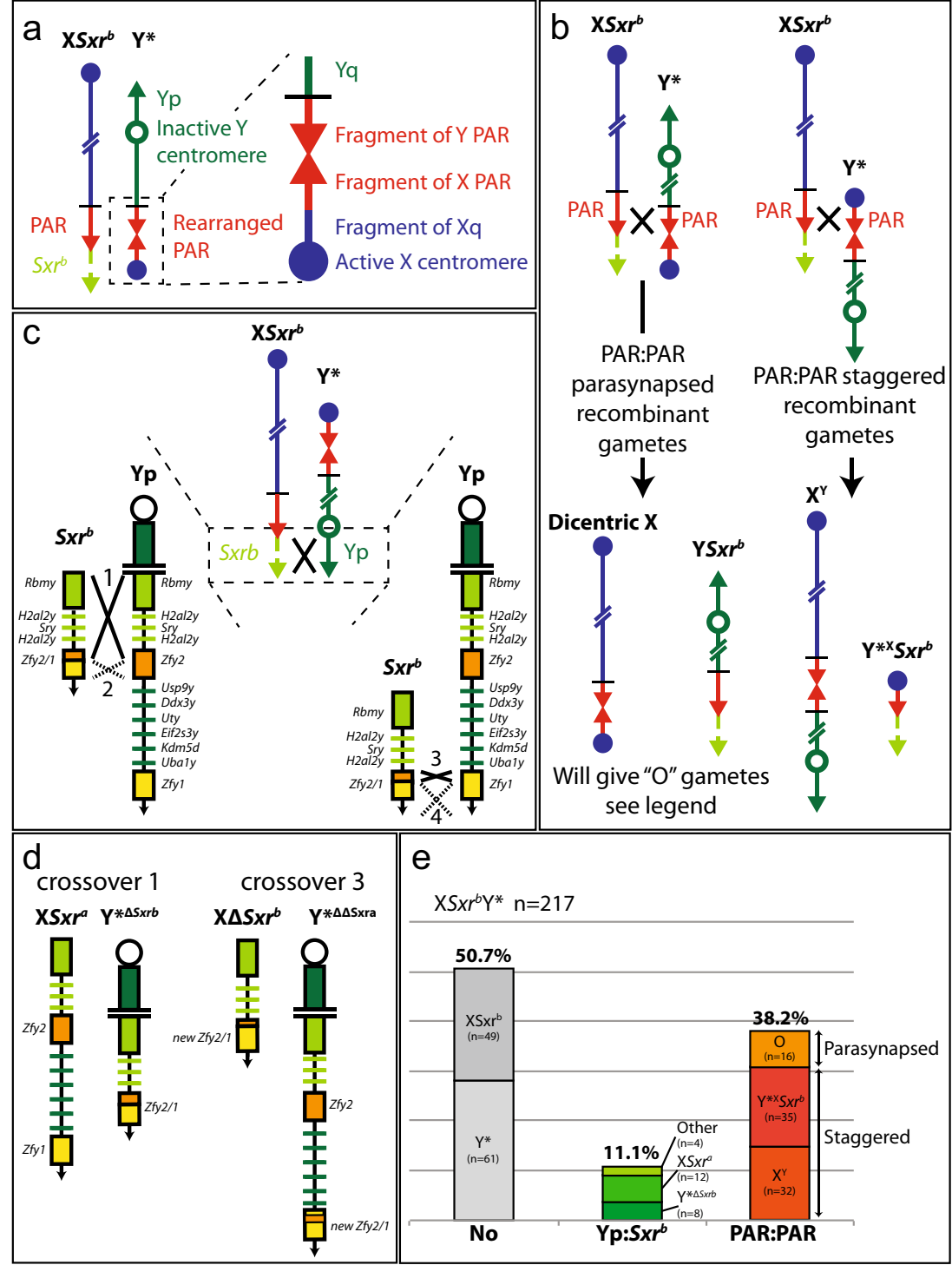

4. PCR X vs Yp To double-check some genotyping, we performed a qualitative ratio measurement of the $\mathrm{X}$ and $\mathrm{Yp} /$ $\mathrm{Sxr}^{\mathrm{a}}$ fragments. Primers o4110 and o4111 (see Table 2) amplify a 161-bp fragment of X-linked $D d x 3 x$ and a 128bp fragment of Yp-linked $D d x 3 y$. The difference in band intensity gives information about the $\mathrm{X}$ vs $\mathrm{Yp} / S x r^{a}$ dose.

5. PCR new $\mathrm{Sxr}^{b}$ To confirm the products of crossover 3 $\left(\mathrm{X} \Delta S x r^{b}\right.$ and $\mathrm{Y}^{\Delta \Delta \mathrm{Sxra}}$ ) that creates a new $Z f y 2 / 1$ fusion gene (Supplemental Fig. S1), we have designed several PCR/RFLP tests allowing amplification of both $Z f y l$ and Zfy2 but, thanks to specific SNPs, digestion of only one (primers o001/o002, o003/o004, o005/o006, and o021/o022).

6. $Y^{* X}{ }_{q P C R}$ (Vernet et al. 2014). To detect the presence of $\mathrm{Y}^{*} \mathrm{X}$, we utilized qPCRs for X-linked $\operatorname{Pr} d x 4$ (absent in $\mathrm{Y}^{* \mathrm{X}}$ ), Amelx (present in $\mathrm{Y}^{* \mathrm{X}}$ ) and Myog (on chromosome 1) for normalization.

\section{Immunostaining to identify X-Y bivalent configurations}

In $\mathrm{XY}$ males, the frequency of $\mathrm{X}$ and $\mathrm{Y}$ univalence at pachytene is $<5 \%$ but it is usually in excess of $20 \%$ when $S x r$ is present. In an extensive study involving males with $S x r$ attached to the $\mathrm{X}$ and/or Y PAR, the $\mathrm{X}$ and $\mathrm{Y}$ were separated in 26.5-86.9 \% of pachytene spermatocytes as compared to $3.5 \%$ in $\mathrm{XY}$ controls (Tease and Cattanach 1989). However, in the absence of synapsis, no crossover can form, and the resulting univalence at the first meiotic metaphase (MI) leads to a $~ 97 \%$ efficient elimination of the spermatocytes by the MI spindle checkpoint (Burgoyne et al. 1992, 2009; Sutcliffe and Burgoyne 1989; Vernet et al. 2011, 2014). Thus, these spermatocytes with $\mathrm{X}$ and $\mathrm{Y}$ univalence make a negligible contribution to the offspring. Our interest was therefore restricted to pachytene spermatocytes with X-Y bivalents. 
Table 1 Screening for recombinant offspring from $\mathrm{XSx} r^{b} \mathrm{Y}$ and $\mathrm{XS} x r^{b} \mathrm{Y}^{*}$ matings

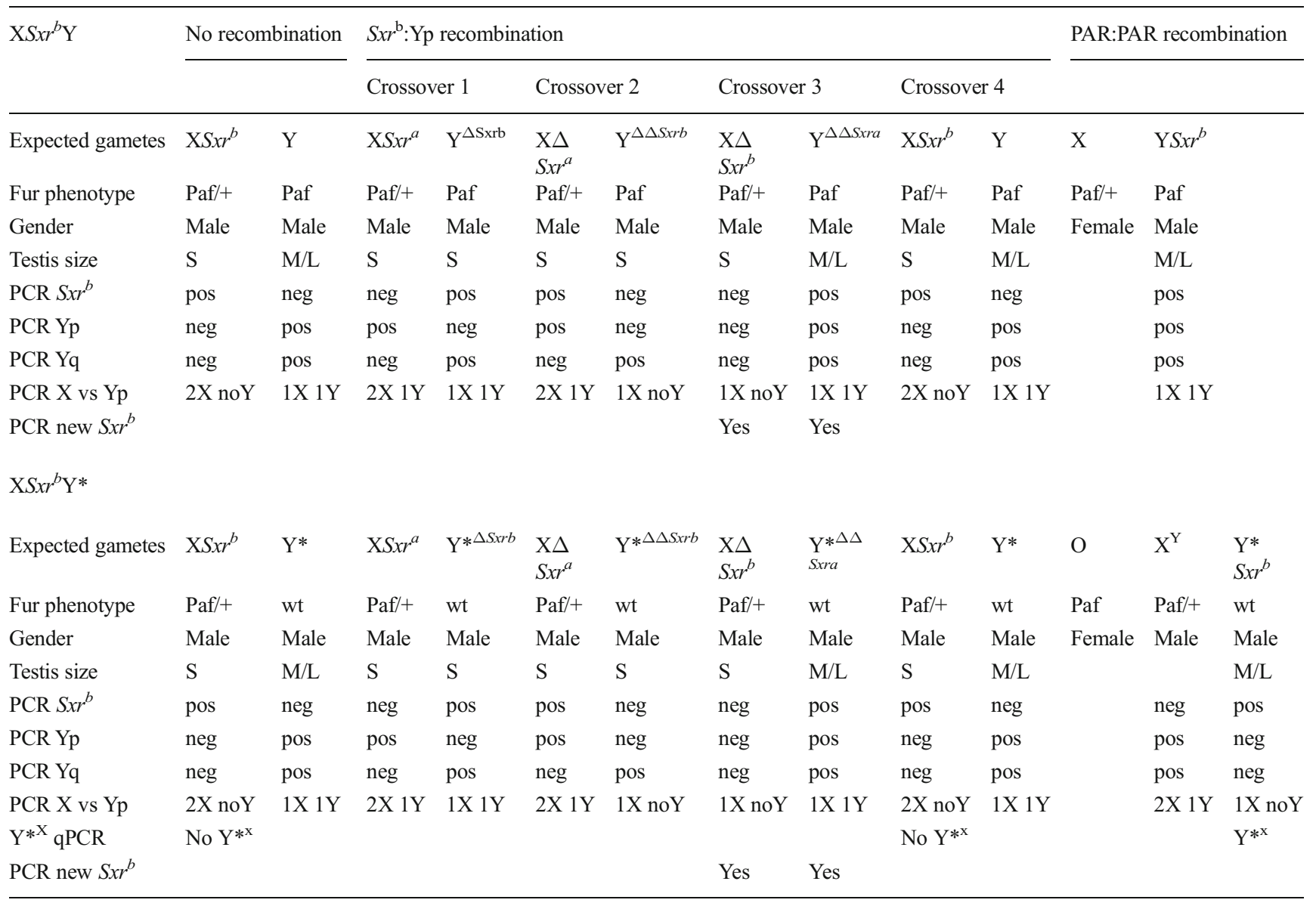

$S$ small, $M$ medium, $L$ large, pos positive, neg negative

To investigate the $\mathrm{X}-\mathrm{Y}$ bivalent configurations, we used immunostaining of surface spread spermatocytes as previously described (Turner et al. 2004) using the following antibodies: Guinea pig polyclonal anti-SCP3 (dilution 1:400, gift from James M Turner), mouse monoclonal anti- $\gamma \mathrm{H} 2 \mathrm{AX}$ (dilution 1:100, Millipore cat. No. 05-636), Human anti-CREST (dilution 1:50, gift from William Earnshaw). Images were captured on an Olympus IX70 inverted microscope. Each fluorochrome image was captured separately as a 12-bit source image by using a computer-assisted (Deltavision) liquid-cooled CCD (Photometrics CH350L; Sensor: Kodak KAF1400, 1317×1035 pixels). A single multiband dichroic mirror was used to eliminate shifts between different filters. Captured images were processed with Fiji and Adobe Photoshop CS5.1.

\section{Yp:Sxr $r^{b}$ recombination crossover sizes estimation and location of potential DSB hotspots}

Size estimates of Yp, the $S x r^{a}$ region, and the sub regions flanking the $S x r^{b}$ deletion breakpoints are based on the most recent published sequence of the mouse Y chromosome (Soh et al. 2014 File 3 in Data S1) and the sequence of the $S x r^{b}$ deletion breakpoint (Decarpentrie et al. 2012). They are minimum size estimates as the Yp sequence is not yet complete. Three large blocks of N's within the Yp sequence, totalling $450 \mathrm{~kb}$, were excluded.

The $S x r^{a}$ breakpoint has not been sequenced but is known to fall within the distal end of the 37-kb Rbmy-repeat tandem array. Based on the recent estimate of 30 Rbmy copies in Yp (Soh et al. 2014) and our previous estimate that 1 in 7 Rbmyrepeats remain in $\mathrm{Sxr}^{a}$ (Mahadevaiah et al. 1998), we have assumed that the $S x r^{a}$ breakpoint lies between the fourth and fifth most distal copies of the Rbmy-repeat array.

The Yp estimate was derived from the sequence telomeric to the centromeric heterochromatin, the latter defined as bases 57238-147035 of Genbank AC175459. The Soh et al. sequence includes 10 of the estimated 30 Rbmy repeats and we therefore added $740 \mathrm{~kb}$ to the Soh et al. sequence length (20 times a repeat unit length of $37 \mathrm{~kb}$ ).

The Soh et al. sequence does not include the Prssly or Teyorf1 genes, which we have mapped to the $S x r^{b}$ region (unpublished data). These two genes are present in an isolated BAC sequence of $185 \mathrm{~kb}$ (NW_001034423). We have assumed that Prssly and Teyorfl are located distal to Zfyl since there is no gap in the Soh 


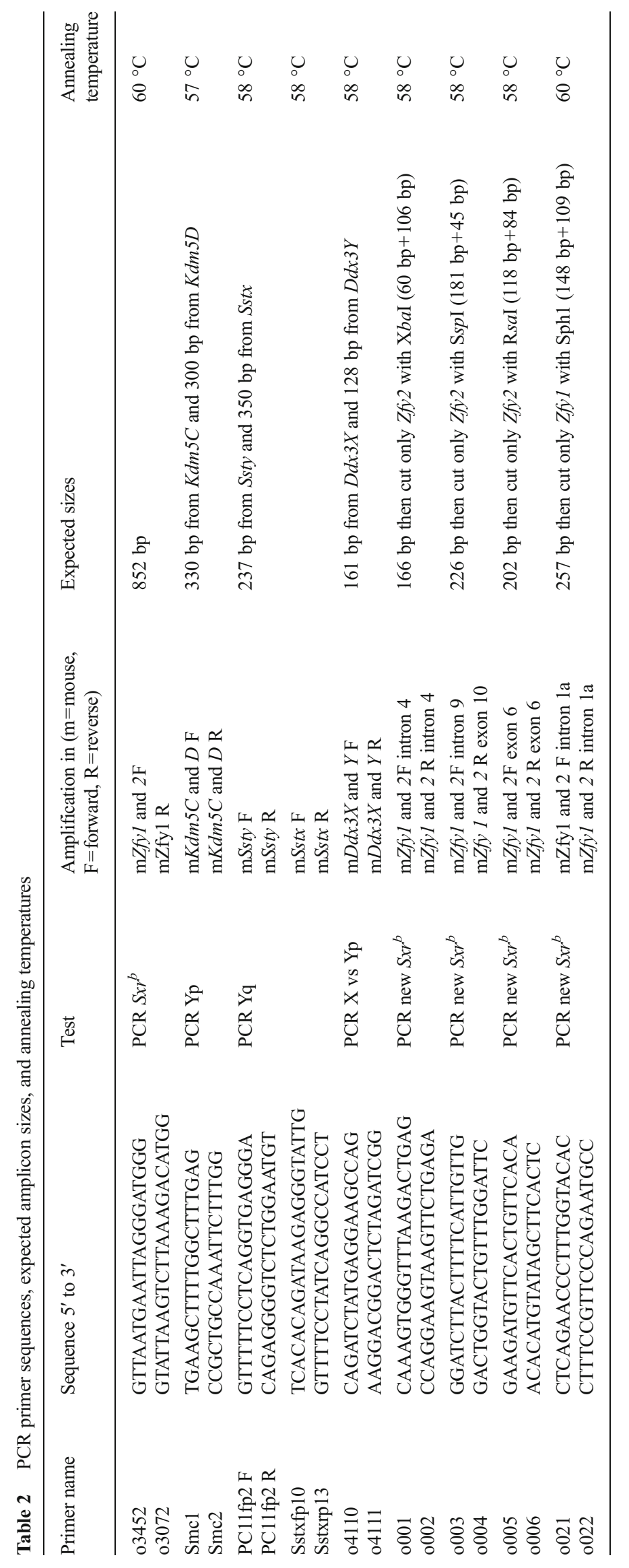


et al. sequence between $Z f y 1$ and the $S x r^{a}$ breakpoint. We therefore added $185 \mathrm{~kb}$ to estimates based on the Soh et al. sequence for Yp and the distance from the telomere to the $S x r^{b}$ breakpoint.

Sixteen potential DSB hotspots have been identified in Yp (Brick et al. 2012; Supplementary Data File 1: List of DSB hotspots and $\mathrm{H} 3 \mathrm{~K} 4 \mathrm{me} 3$ marks). To determine which of these are located within $S x r^{b}$, the sequence coordinates were used to identify the associated DNA sequences in the mouse genome using NCBI37/mm9 (as used by Brick et al. 2012), where necessary checking these against the sequence data we have deduced for $\mathrm{Sxr}^{b}$ based on the data from Soh et al. 2014.

\section{Results and discussion}

The recombination data we present here were obtained from PCR genotyping of offspring (see Materials and Methods) that derive from two crosses. Firstly, there are data for 160 offspring obtained from $\mathrm{XSxr}{ }^{b} \mathrm{Y} \times \mathrm{XX}$ matings. Secondly, there are data for 217 offspring from $\mathrm{XSxr}^{a} \mathrm{Y}^{*} \times \mathrm{XX}$ matings.

\section{Recombination in $\mathrm{XSxr}^{\mathrm{b}} \mathrm{Y}$}

As expected, PAR-PAR recombination produces $\mathrm{X}$ and $\mathrm{YSxr}{ }^{b}$ recombinants (52.5\% of offspring). Yp- $S x r^{b}$ recombination produced the recombinants $\mathrm{XSxr}^{\mathrm{a}}$ and $\mathrm{Y}^{\Delta S x r b}(5.6 \%)$ that are predicted for crossover 1 (Fig. 2a, b, c). Recombinants were not seen for the potential crossover 2 nor were there recombinants deriving from the potential alternative synaptic alignment illustrated in Fig. 3c. Overall, there was a deficiency of non-recombinants $(n=67,41.9 \%)$ as compared to recombinants $(n=93,58.1 \%)$, and this was just significantly different from the expected $50 \%$ of each [Binomial test (http://www.measuringu.com/onep.php); two sided $p$ value $=$ 0.0478]. We have no explanation for this deficiency, and it was not observed with the larger sample of data for the $\mathrm{XSxr}^{b} \mathrm{Y}^{*}$ model (see below).

\section{Recombination in $\mathrm{XSxr}^{\mathrm{b}} \mathrm{Y}^{*}$}

The $\mathrm{Y}^{*}$ chromosome has a complex PAR involving duplication and deletion of PAR DNA sequences and an X-derived centromere distal to this complex PAR (Fig. 3a) (Burgoyne and Evans 2000; Burgoyne et al. 1998; Eicher et al. 1991; Rodriguez and Burgoyne 2001). This leads to two orientations for PAR-PAR synapsis and to the production of four distinct recombinant chromosomes following PAR recombination (Fig. 3b). The opportunities for $\mathrm{Yp}-\mathrm{Sxr}^{b}$ synapsis (Fig. 3c) are the same as those in $\mathrm{XSxr}{ }^{b} \mathrm{Y}$. Four types of recombinant arising from crossovers 1 (20 recombinants) and 3 (4 recombinants) were identified (Fig. 3d). The predominance of recombinants involving crossover 1 is to be expected given that it covers $780 \mathrm{~kb}$, which is more than 25 -fold longer than for crossovers 2 and 3 (see Table 3 ). Crossover 4 does cover $580 \mathrm{~kb}$, but we have no markers to detect recombination in this region. The frequencies of the various recombinants identified among the 217 offspring of $\mathrm{XSxr}^{b} \mathrm{Y}^{*}$ males are illustrated in Fig. 3e.

How do the recombination data for $\mathrm{XSxr}^{b} \mathrm{Y}^{*}$ males compare with those of $\mathrm{XSxr}^{b} \mathrm{Y}$ males? As expected, the $\mathrm{XSxr}^{b} \mathrm{Y}^{*}$ PAR-PAR recombinants differ due to the presence of the $\mathrm{Y}^{*}$ chromosome. There is no deficiency in the number of nonrecombinants $(n=110,50.7 \%)$ relative to recombinants $(n=$ $107,49.3 \%$ ), but there is an increase in frequency of $\mathrm{Yp}-\mathrm{Sx} r^{b}$ relative to PAR-PAR recombinants in $\mathrm{XSxr}^{b} \mathrm{Y}^{*}(11.1 \%, n=$ 24 vs 83$)$ as compared to $\mathrm{XSxr}{ }^{b} \mathrm{Y}(5.6 \%, n=9$ vs 84$)(P=0.02$ Fisher's exact test, two-tailed). These recombinants include four with exchanges that are generated by crossover 3 . The rare recombinants due to crossover 3 were not identified in $\mathrm{XSxr}{ }^{b} \mathrm{Y}$, but this may just reflect the smaller sample size (9 vs 24).

Table 3 Approximate sizes of PAR, Yp, $S x r^{b}$, and of potential crossover regions

\begin{tabular}{|c|c|c|}
\hline & Areas covered & Homology size regions \\
\hline Pseudo autosomal region (PAR) & & $\approx 700 \mathrm{~kb}^{\mathrm{a}}$ \\
\hline Short arm of the Y (Yp) & From 30 copies Rbmy to distal end of Yp & $4 \mathrm{Mb}$ \\
\hline$S x r^{a}$ domain & From 4 copies Rbmy to distal end of Yp & $2.24 \mathrm{Mb}$ \\
\hline$S x r^{b}$ domain & From 4 copies Rbmy, 2 copies H2al2y, Sry, Zfy2/1 to distal end of Yp & $860 \mathrm{~kb}$ \\
\hline$\Delta^{S x r b}$ deletion & From intron $4 Z f y 2$ to intron $4 Z f y 1$ & $1.38 \mathrm{Mb}$ \\
\hline \multicolumn{3}{|l|}{ Yp:Sxr ${ }^{b}$ recombination crossover } \\
\hline Crossover 1 & From 5 copies $R b m y$ to $S x r^{b}$ break point & $780 \mathrm{~kb}$ \\
\hline Crossover 2 & Between $Z f y 1$ part of $Z f y 2 / 1$ and $Z f y 2$ & $29 \mathrm{~kb}^{\mathrm{b}}$ \\
\hline Crossover 3 & Between $Z f y 2$ part of $Z f y 2 / 1$ and $Z f y 1$ & $21 \mathrm{~kb}^{\mathrm{b}}$ \\
\hline Crossover 4 & From $Z f y 1$ part of $Z f y 2 / 1$ to distal end of $Y p$ & $580 \mathrm{~kb}$ \\
\hline
\end{tabular}

${ }^{\text {a }}$ Perry et al. 2001

${ }^{\mathrm{b}}$ Maximum crossover size between $Z f y 1$ and $Z f y 2$ with an average of $90 \%$ of homology 
Is $\mathrm{Yp}-\mathrm{Sxr}{ }^{\mathrm{b}}$ recombination potentiated by the attachment of $S x r^{b}$ to the X PAR?

The mouse PAR is estimated to be only $\sim 700 \mathrm{~kb}$ (Perry et al. 2001). Nevertheless, one or two SPO11-mediated DSBs are regularly generated in the $\mathrm{X}$ and $\mathrm{Y}$ PARs (albeit later than those on autosomes); these DSBs reliably drive the synapsis and formation of a crossover necessary to ensure $\mathrm{X}$ and $\mathrm{Y}$ segregation at MI (Kauppi et al. 2011, 2012). Yp in normal males is largely protected from recombination because SPO11-mediated DSBs are not preferentially targeted to this region and because it lacks homology with the DSB rich $\mathrm{X}$ and Y PARs.

In males with $S_{x r^{b}}$ attached to the X PAR, there are two reasons why the $\mathrm{Yp}-S x r^{b}$ recombination is likely to be preferentially initiated by DSBs in $S x r^{b}$. Firstly, we estimate that $S x r^{b}$ is $860 \mathrm{~kb}$, whereas Yp we estimate to be $4 \mathrm{Mb}$; this is due to the $1.38-\mathrm{Mb} S x r^{b}$ interstitial deletion ( $\Delta^{S x r b}$ in Fig. 1d) and the reduced number of copies of Rbmy (Mahadevaiah et al. 1998). Thus, $3.14 \mathrm{Mb}$ of Yp has no homology to $S x r^{b}$ so that DSBs located in this $3.14 \mathrm{Mb}$ will not be able to find a homologous partner during the presynaptic homology search. On the other hand, with a size of $860 \mathrm{~kb}, S x r^{b}$ DNA will only very rarely be cut by Spo11 unless this region has one or more DSB hotspots. This led us to hypothesize that $S x r^{b}$ may have DSB hotspots because it is attached to the DSB-rich X PAR. Support for this hypothesis is provided by DSB mapping data (Brick et al. 2012). It has been established that the majority of DNA hotspots co-localize with the histone H3 methyl transferase PRDM9, and these authors found that the $\mathrm{X}$ PAR together with $\sim 900 \mathrm{~kb}$ of upstream X-specific DNA is unique in having PRDM9-independent DSB hotspots (hotspots that remain in $\operatorname{Prdm} 9$ knockout mice). The frequency of these hotspots declines with increasing distance from the $\mathrm{X}$ PAR boundary. We therefore suggest that when $S x r^{b}$ is attached to the distal end of the X PAR, it is similarly bestowed with DSB hotspots that decrease in frequency with increasing distance from the PAR. If this is true, the highest density of DSBs should be in the region covered by crossover 1 , reducing to a minimum in the region covered by crossover 4. However, if during the homology search, single-stranded DSB tails originating in the region covered by crossovers 3-4 do successfully invade the homologous sequences in $\mathrm{Yp}$, then the resulting synaptic alignment may inhibit exchanges in the region covered by crossovers 1 and 2 . We have not identified any double recombinants with PAR-PAR and $S x r^{b}-Y p$ exchanges; this is presumably a reflection of the low likelihood that both regions of homology are juxtaposed during the presynaptic homology search.
Also of potential relevance to the location of DSBs within $S_{x r} r^{b}$ are the 16 potential DSB hotspots identified in Yp (Brick et al. 2012; Supplementary Data File 1: List of DSB hotspots and H3K4me3 marks). However, only one (chrY:2158709-2159150) is retained in $S x r^{b}$. This is located within each of the few Rbmy genes that remain close to the $S x r^{b}$ breakpoint that abuts the distal end of the PAR.

\section{Presynaptic telomere congregation and the incidence of $\mathrm{Yp}-\mathrm{Sx} \mathrm{r}^{\mathrm{b}}$ recombination}

In the $1970 \mathrm{~s}$, Solari carried out painstaking electron microscopic reconstructions of the $\mathrm{X}-\mathrm{Y}$ bivalent at pachytene that served to identify the juxtaposition of what we now know to be the X and Y PAR telomeric ends at the nuclear membrane attachment site and the presence of a synaptonemal complex between the PAR axes; the non PAR ends of the X-Y bivalent although attached to the nuclear membrane were not closely associated (Solari 1970, 1974). The association of the PAR ends is now widely accepted to be initiated prior to synapsis by the congregation of the telomeric ends of all chromosomes during zygotene (bouquet formation), together with dynamic chromosome movements during the bouquet phase that can bring homologous segments in sufficiently close proximity to enable homologous DNA strand invasion and synapsis (Scherthan 2001; Shibuya et al. 2014).

How might the telomeric congregation and homology recognition phases be affected in $\mathrm{XS} x r^{b} \mathrm{Y}$ and $\mathrm{XS} x r^{b} \mathrm{Y}^{*}$ males? In $\mathrm{X} S x r^{b} \mathrm{Y}$ the $\mathrm{XS} x r^{b}$, telomere and adjacent $S x r^{b}$ DNA sequences match those of Yp rather than those of $Y$ PAR. This will provide the opportunity for homology recognition and synapsis of $S x r^{b}$ with Yp-examples of such bivalents are seen in pachytene spermatocyte spreads (Fig. 4a). Nevertheless, the majority of synaptic associations involve the X PAR/Sxr $r^{b}$ and Y PAR ends (Fig. 4b) and the recombination data document frequent PAR-PAR recombination (Fig. 2c). This is unsurprising since the $S x r^{b}$ segment is short $(860 \mathrm{~kb})$ so that the $\mathrm{X}$ and Y PARs are still close to the membrane attachment sites where the dynamic chromosome movements during the bouquet phase could also lead to PAR-PAR homology recognition.

In $\mathrm{XS} x r^{b} \mathrm{Y}^{*}$ the PAR/Sxr ${ }^{b}$ and Yp, telomeric ends are the same as those of $\mathrm{XSx} r^{b} \mathrm{Y}$ thus enabling homology recognition and crossing over. However, there is one feature of $\mathrm{Y}^{*}$ that might increase the frequency of $\mathrm{Yp}-\mathrm{S} x r^{b}$ exchanges relative to PAR-PAR exchanges. The PAR end of $\mathrm{Y}^{*}$ terminates in an $\mathrm{X}$-derived centromeric region that includes some copies of a multi copy $\mathrm{X}$ sequence DXHXF34 (Laval et al. 1997; Rodriguez and Burgoyne 2001) that derives from a multi copy $X$ gene that we have termed Sstx (Touré et al. 2004); recent sequence 
Fig. 4 X-Y bivalent configurations at pachytene in $\mathrm{XSx} r^{b} \mathrm{Y}$ and $\mathrm{XSx} r^{b} \mathrm{Y}^{*}$ males. Each panel has a diagram of the predicted crossover event, a low magnification view showing the $\mathrm{X}$-Y bivalent in a $\gamma \mathrm{H} 2 \mathrm{AX}$-stained (red) sex chromatin cloud together with near-by autosomes, a higher power view of the X-Y bivalent with the $\gamma \mathrm{H} 2 \mathrm{AX}$ staining removed, and a drawing of the deduced X-Y bivalent configuration highlighting the centromere positions. Note that the red CREST staining of active centromeres often appears as yellow where it overlaps the green chromosome axis. (a) $\mathrm{XSxr}{ }^{b} \mathrm{Y}$ with $\mathrm{Yp}-\mathrm{Sxr}^{b}$ synapsis. (b) $\mathrm{XS} x r^{b} \mathrm{Y}$ with PAR-PAR synapsis. (c) $\mathrm{XSxr}{ }^{b} \mathrm{Y}^{*}$ with centromerecentromere association - this is likely to be due to synapsis driven by homology of Sstx sequences adjacent to the $\mathrm{X}$ and $\mathrm{Y}^{*}(\mathrm{X}$-derived) centromeres. (d) $\mathrm{XSxr}^{b} \mathrm{Y}^{*}$ with Yp-Sxr ${ }^{b}$ synapsis. (e) XSxr ${ }^{b-}$ $\mathrm{Y}^{*}$ with centromere-centromere and $\mathrm{Yp}-\mathrm{S} x r^{b}$ synapsis. (f) $\mathrm{XS} x r^{b-}$ $\mathrm{Y}^{*}$ with PAR-PAR synapsisparasynapsis and staggered synapsis cannot be differentiated at this resolution. The staggered configuration is indicated in the black square

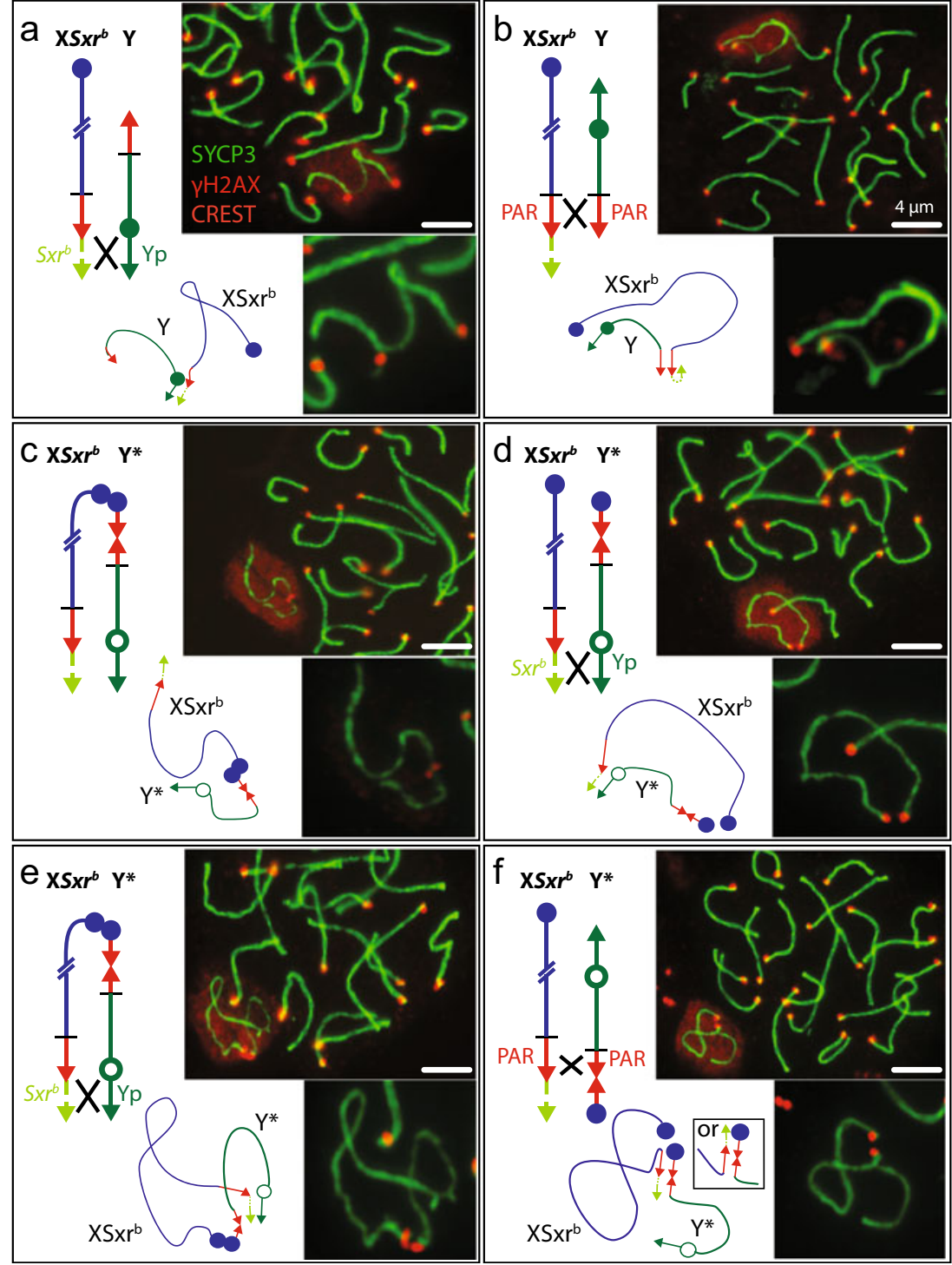

information from Soh et al. (2014 - see footnote to their Figure 5) has indicated that the majority of copies of Sstx are located adjacent to the $\mathrm{X}$ centromere. This is expected to promote homology recognition and synapsis of the $\mathrm{PAR} /$ centromeric end of $\mathrm{Y}^{*}$ with the centromeric end of the $\mathrm{X}$ chromosome-examples of this can be seen in pachytene spermatocyte spreads (Fig. 4c). This could lead to crossing over adjacent to the centromeres within the Sstx repeat but we have not attempted to detect this. We identified three other pachytene configurations that we interpret as resulting from Yp-Sxr ${ }^{b}$ synapsis (Fig. 4d), $\mathrm{Yp}-\mathrm{Sxr}^{b}$ synapsis and $\mathrm{X}$ centromere- $\mathrm{Y}^{*} \mathrm{PAR} /$ centromere synapsis (Fig. 4e) and PAR-PAR synapsis (Fig. 4f). We therefore wondered whether $\mathrm{Y}^{*}$ promotion of alternative synaptic configurations to PAR-PAR synapsis might be responsible for the significant increase in the frequency of $\mathrm{Yp}-S x r^{b}$ recombinants relative to PAR-PAR recombinants. In order to test this, we classified the synaptic configurations of 99 pachytene spermatocyte spreads from each genotype, which yielded the following results: $\mathrm{XSxr} r^{b} \mathrm{Y}-78$ PAR-PAR, 21Yp-Sxr $r^{b}$ XS $x r^{b} \mathrm{Y}^{*}-77$ PAR-

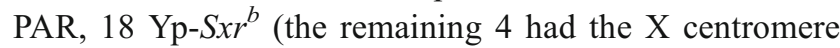
associated with $\mathrm{X}$-derived centromere of $\mathrm{Y}^{*}$ as in Fig. 4c). From this, it is clear that the presence of $\mathrm{Y}^{*}$ is not promoting $\mathrm{Yp}-\mathrm{S} x r^{b}$ synapsis at the expense of PARPAR synapsis. A plausible alternative explanation is that synapsis of the complex $\mathrm{Y}^{*}$ PAR with the X PAR is less likely to result in a crossover because in either the staggered or parasynapsed orientations there is a reduced PAR segment available for homologous synapsis. In those that fail to form a crossover, the $\mathrm{X}$ and $\mathrm{Y}$ will separate at the end of prophase, be subject to SAC-triggered apoptotic elimination at MI and will thus not contribute to the progeny (Burgoyne et al. 2009; Vernet et al. 2011, 2014). 


\section{Conclusions}

The present findings documenting frequent recombination between the Y chromosome short arm (Yp) and the Yp-derived $\mathrm{Sxr}^{b}$ chromosome segment attached distal to the $\mathrm{X}$ chromosome PAR, confirm and extend the findings of work published in 1984-1995 (Epplen et al. 1988; Laval et al. 1995; McLaren et al. 1988, 1992; Simpson et al. 1984). Placing these findings in the context of current views as to the transition from meiotic DSB formation through homology search to synapsis has enabled us to propose a model to explain this frequent $\mathrm{Yp}-\mathrm{Sxr} r^{b}$ recombination. A key issue is the location of the DSBs that generate the single-stranded DNA tail that executes the homology search leading to synapsis between Yp and $S x r^{b}$. In normal $\mathrm{XY}$ males, DSBs are absent (or extremely rare) at the non-PAR end of the Y. Instead, 1-2 DSBs are directed to the Y PAR; the X PAR also has 1-2 PAR DSBs (Kauppi et al. 2011, 2012). Importantly, DSB mapping has established that there is a spreading of DSB hotspot activity from the X PAR into $\sim 900 \mathrm{~kb}$ of adjacent X-specific DNA (Brick et al. 2012). We now propose that DSB hotspot activity also spreads from the distal end of the $\mathrm{X}$ PAR into $S x r^{b}$, thus potentiating exchanges with Yp. The next issue is whether there is any likelihood that the single-stranded DNA tails generated within $\mathrm{Sxr}^{b}$ will come close enough to Yp to enable homology recognition and synapsis. We propose that this is likely because the $S x r^{b}$ and Yp ends of the chromosomes will be located at nuclear membrane attachment sites, so that telomere congregation and the associated dynamic movement of the clustered chromosome ends during zygotene will promote their interaction (Scherthan 2001; Shibuya et al. 2014). It is very reassuring that the $\mathrm{Yp}-\mathrm{Sxr}^{b}$ recombination in these unusual mouse models fits so comfortably with current views as to how homologous synapsis is achieved.

Acknowledgments NIMR Biological Services provided help with the mouse breeding, and Áine Rattigan provided valuable help with the PCR genotyping.

Compliance with ethical standards Laboratory mice were exclusively used for this study, and all applicable international, national, and institutional guidelines for the care and use of animals were followed.

Conflict of interest All authors declare that they have no competing interests.

Funding The work was funded by the Medical Research Council UK ([U117532009 to P.S.B., a Career Development Fellowship to F.D.).

Open Access This article is distributed under the terms of the Creative Commons Attribution 4.0 International License (http:// creativecommons.org/licenses/by/4.0/), which permits unrestricted use, distribution, and reproduction in any medium, provided you give appropriate credit to the original author(s) and the source, provide a link to the Creative Commons license, and indicate if changes were made.

\section{References}

Brick K, Smagulova F, Khil P, Camerini-Otero RD, Petukhova GV (2012) Genetic recombination is directed away from functional genomic elements in mice. Nature 485:642-645. doi:10.1038/nature11089

Burgoyne PS (1982) Genetic homology and crossing over in the $\mathrm{X}$ and $\mathrm{Y}$ chromosomes of mammals. Hum Genet 61:85-90

Burgoyne PS, Evans EP (2000) A high frequency of XO offspring from $\mathrm{X}(\mathrm{Paf}) \mathrm{Y}^{*}$ male mice: evidence that the Paf mutation involves an inversion spanning the X PAR boundary. Cytogenet Cell Genet 91:57-61

Burgoyne PS, Mahadevaiah SK, Sutcliffe MJ, Palmer SJ (1992) Fertility in mice requires $\mathrm{X}-\mathrm{Y}$ pairing and a Y-chromosomal "spermiogenesis" gene mapping to the long arm. Cell 71:391-398

Burgoyne PS, Mahadevaiah SK, Perry J, Palmer SJ, Ashworth A (1998) The $\mathrm{Y}^{*}$ rearrangement in mice: new insights into a perplexing PAR. Cytogenet Cell Genet 80:37-40

Burgoyne PS, Mahadevaiah SK, Turner JM (2009) The consequences of asynapsis for mammalian meiosis. Nat Rev Genet 10:207-216

Cattanach BM, Evans EP, Burtenshaw MD, Barlow J (1982) Male, female and intersex development in mice of identical chromosome constitution. Nature 300:445-446

Decarpentrie F et al (2012) Human and mouse ZFY genes produce a conserved testis-specific transcript encoding a zinc finger protein with a short acidic domain and modified transactivation potential. Hum Mol Genet 21:2631-2645. doi:10.1093/hmg/dds088

Eicher EM et al (1991) The mouse $\mathrm{Y}^{*}$ chromosome involves a complex rearrangement, including interstitial positioning of the pseudoautosomal region. Cytogenet Cell Genet 57:221-230

Epplen JT, Studer R, McLaren A (1988) Heterogeneity in the Sxr (sexreversal) locus of the mouse as revealed by synthetic GATA-GACA probes. Genet Res 51:239-246

Kauppi L, Jasin M, Keeney S (2012) The tricky path to recombining X and Y chromosomes in meiosis. Ann N Y Acad Sci 1267:18-23. doi:10.1111/j.1749-6632.2012.06593.x

Kauppi L, Barchi M, Baudat F, Romanienko PJ, Keeney S, Jasin M (2011) Distinct properties of the XY pseudoautosomal region crucial for male meiosis. Science 331:916-920. doi:10.1126/science. 1195774

Lane PW, Davisson MT (1990) Patchy fur (Paf), a semidominant Xlinked gene associated with a high level of X-Y nondisjunction in male mice. J Hered 81:43-50

Lange $\mathrm{J}$ et al (2009) Isodicentric $\mathrm{Y}$ chromosomes and sex disorders as byproducts of homologous recombination that maintains palindromes. Cell 138:855-869

Lange J et al (2013) Intrachromosomal homologous recombination between inverted amplicons on opposing Y-chromosome arms. Genomics 102:257-264. doi:10.1016/j.ygeno.2013.04.018

Laval SH et al (1995) Y chromosome short arm-Sxr recombination in $\mathrm{XSxr} / \mathrm{Y}$ males causes deletion of Rbm and XY female sex reversal. Proc Natl Acad Sci U S A 92:10403-10407

Laval SH, Reed V, Blair HJ, Boyd Y (1997) The structure of DXF34, a human X-linked sequence family with homology to a transcribed mouse Y-linked repeat. Mamm Genome 8:689-691

Mahadevaiah SK et al (1998) Mouse homologues of the human AZF candidate gene RBM are expressed in spermatogonia and spermatids, and map to a Y chromosome deletion interval associated with a high incidence of sperm abnormalities. Hum Mol Genet 7:715-727

Mazeyrat S et al (1998) The mouse Y chromosome interval necessary for spermatogonial proliferation is gene dense with syntenic homology to the human AZFa region. Hum Mol Genet 7:1713-1724

McLaren A, Monk M (1982) Fertile females produced by inactivation of $X$ chromosome of 'sex-reversed' mice. Nature 300:446-448

McLaren A, Simpson E, Epplen JT, Studer R, Koopman P, Evans EP, Burgoyne PS (1988) Location of the genes controlling H-Y antigen 
expression and testis determination on the mouse $\mathrm{Y}$ chromosome. Proc Natl Acad Sci U S A 85:6442-6445

McLaren A, Simpson E, Bishop CE, Mitchell MJ, Darling SM (1992) Recombination between the $\mathrm{X}$ and $\mathrm{Y}$ chromosomes and the Sxr region of the mouse. Genet Res Camb 60:175-184

Moens PB, Chen DJ, Shen Z, Kolas N, Tarsounas M, Heng HHQ, Spyropolous B (1997) Rad51 immunocytology in rat and mouse spermatocytes and oocytes. Chromosoma 106:207-215

Perry J, Palmer S, Gabriel A, Ashworth A (2001) A short pseudoautosomal region in laboratory mice. Genome Res 11: $1826-1832$

Plug AW, Peters AH, Keegan KS, Hoekstra MF, de Boer P, Ashley T (1998) Changes in protein composition of meiotic nodules during mammalian meiosis. J Cell Sci 111(Pt 4): 413-423

Rodriguez TA, Burgoyne PS (2001) Spermatogenic failure in male mice with four sex chromosomes. Chromosoma 110:124-129

Royo $\mathrm{H}$ et al (2010) Evidence that meiotic sex chromosome inactivation is essential for male fertility. Curr Biol 20:2117-2123. doi:10.1016/ j.cub.2010.11.010

Scherthan H (2001) A bouquet makes ends meet. Nat Rev Mol Cell Biol 2:621-627

Shibuya H, Morimoto A, Watanabe Y (2014) The dissection of meiotic chromosome movement in mice using an in vivo electroporation technique. PLoS Genet 10:e1004821. doi:10.1371/journal.pgen. 1004821

Simpson EM, Page DC (1991) An interstitial deletion in mouse Y chromosomal DNA created a transcribed Zfy fusion gene. Genomics 11: 601-608

Simpson E, McLaren A, Chandler P, Tomonari K (1984) Expression of $\mathrm{H}-\mathrm{Y}$ antigen by female mice carrying Sxr. Transplantation 37:17-21
Skaletsky H et al (2003) The male-specific region of the human Y chromosome is a mosaic of discrete sequence classes. Nature 423:825837

Soh YQ et al (2014) Sequencing the mouse Y chromosome reveals convergent gene acquisition and amplification on both sex chromosomes. Cell 159:800-813. doi:10.1016/j.cell.2014.09.052

Solari AJ (1970) The spatial relationship of the X and Y chromosomes during meiotic prophase in mouse spermatocytes. Chromosoma 29: 217-236

Solari AJ (1974) The behaviour of the XY pair in mammals. Int Rev Cytol 38:273-317

Sutcliffe MJ, Burgoyne PS (1989) Analysis of the testes of H-Y negative XOSxrb mice suggests that the spermatogenesis gene (Spy) acts during the differentiation of the A spermatogonia. Development 107:373-380

Tease C, Cattanach BM (1989) Sex chromosome pairing patterns in male mice of novel Sxr genotypes. Chromosoma 97:390-395

Touré A, Grigoriev V, Mahadevaiah SK, Rattigan A, Ojarikre OA, Burgoyne PS (2004) A protein encoded by a member of the multicopy Ssty gene family located on the long arm of the mouse $\mathrm{Y}$ chromosome is expressed during sperm development. Genomics 83:140-147

Turner JM et al (2004) BRCA1, histone H2AX phosphorylation, and male meiotic sex chromosome inactivation. Curr Biol 14:21352142

Vernet $\mathrm{N}$ et al (2011) The Y-encoded gene Zfy2 acts to remove cells with unpaired chromosomes at the first meiotic metaphase in male mice. Curr Biol 21:787-793. doi:10.1016/j.cub.2011.03.057

Vernet N, Mahadevaiah SK, Yamauchi Y, Decarpentrie F, Mitchell MJ, Ward MA, Burgoyne PS (2014) Mouse Y-linked Zfy1 and Zfy2 are expressed during the male-specific interphase between meiosis I and meiosis II and promote the 2nd meiotic division. PLoS Genet 10: e1004444. doi:10.1371/journal.pgen.1004444 